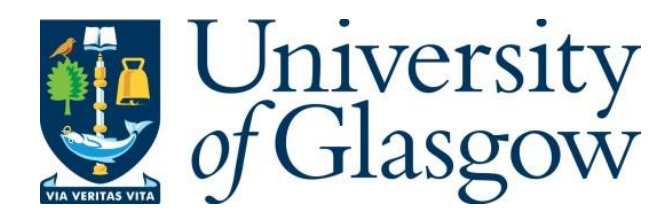

Law, T.K., Lim, F., Li, Y., Teo, J.W. R. and Wei, S. (2017) Effects of Humidity on the Electro-Optical-Thermal Characteristics of High-Power LEDs. In: 2016 IEEE 18th Electronics Packaging Technology Conference (EPTC), Singapore, 30 Nov - 03 Dec 2016, pp. 718-723. ISBN 9781509043705.

There may be differences between this version and the published version. You are advised to consult the publisher's version if you wish to cite from it.

http://eprints.gla.ac.uk/145751/

Deposited on: 11 August 2017

Enlighten - Research publications by members of the University of Glasgow http://eprints.gla.ac.uk 


\title{
Effects of Humidity on the Electro-Optical-Thermal Characteristics of High-Power LEDs
}

\author{
T.K. Law ${ }^{\mathrm{a}, \mathrm{b}}$, Fannon Limª, Y. Li ${ }^{\mathrm{a}}$, J.W.Ronnie Teo ${ }^{\mathrm{a}, \mathrm{c}}$, Sophia Wei ${ }^{\mathrm{b}}$

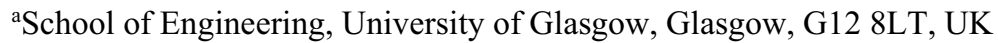 \\ ${ }^{b}$ School of Engineering, Nanyang Polytechnic, 180 Ang Mo Kio Avenue 8, Singapore 569830 \\ 'Singapore Institute of Manufacturing Technology, 71 Nanyang Drive, Singapore 638075
}

\begin{abstract}
LEDs are subjected to environments with high moisture in many applications. In this paper, the experiments reveal photometric and colorimetric degradation at high humidity. Corresponding spectral power analysis and parameter extraction indicate that the flip-chip bonded LED samples show accelerated chip failure compared to the conventionally bonded samples. The chip-related failure induces greater heat accumulation, which correlates with the increase in heating power observed in the package. However, the temperature rise and thermal resistance for the flip-chip bonded LEDs do not increase substantially as compared to the conventionally bonded LEDs. This is because the junction temperature can be reduced with a flip-chip die-bonding configuration where the heat generated in the LED chip is dissipated effectively onto the AlN substrate, thereby reducing the increase in temperature rise and thermal resistance. The experimental results are supported by evaluation of the derivative structure functions. In addition, as the thermal resistance of the LED package varies with different humidity levels, there is a need to specify the conditions of humidity in data sheets as LED manufacturers routinely specify a universal thermal resistance value under a fixed operating condition.
\end{abstract}

\section{Introduction}

There has been much progress in phosphor-converted light emitting diode (pcLED) technology over the last few years. PcLEDs are being used in many commercial applications in diverse environments. Despite common usage in high humidity applications, there are few works on the study of moisture effects on pcLEDs [1].

In high humidity conditions, the optical output degradation is related to both package and chip failure. Moisture diffuses into the interfaces of packaging material which causes a decrease in light output [2] while chip failure causes the blue intensity to degrade faster that the yellow intensity of the optical output [3]. Moisture inside LED packaging may also cause thermal resistance to increase, due to delamination between chip and substrate in high moisture content environments $[4,5]$. This exacerbates the package's thermal load, bringing about a corresponding increase in junction temperature.

This paper focuses on the impact of long term humidity effects on two different types of LEDs based on their diebonding configuration, which is either flip-chip (FC) or conventional (C) bonding. While studies on flip-chip LEDs have demonstrated good optical extraction efficiencies and thermal dissipation properties [6-8], there is a need for research on the performance of flip-chip LEDs under humidity aging compared to LEDs with conventional die-bonding. Through understanding the implications of high moisture environments on the optical and thermal performance of a package, the performance and reliability of high power pcLED packages can be improved.

\section{Experimental Setup and Procedures}

Two types of $1 \mathrm{~W}$ rated pcLED packages with different die-bonding configurations (flip-chip or conventional) are used in this experiment. The LEDs are commercial $1 \mathrm{~mm}^{2}$ GaN LED, with a blue peak wavelength of $\sim 450 \mathrm{~nm}$. A sample size of 20 is used for each package at each humidity level ( $10 \%$ and $85 \%$ relative humidity). The humidity level of $85 \%$ is within the range of daily relative humidity levels in several countries in Southeast Asia [9]. For the C LED package, GaN LED is grown onto sapphire and subsequently bonded onto a heat spreading substrate. The FC LEDs adopt a flip-chip die-bonding approach whereby the GaN LED device is directly bonded onto heat spreading substrate via Au bumps. The micrographs in Fig. 1 illustrate the difference in bonding architecture between these two types of LEDs.

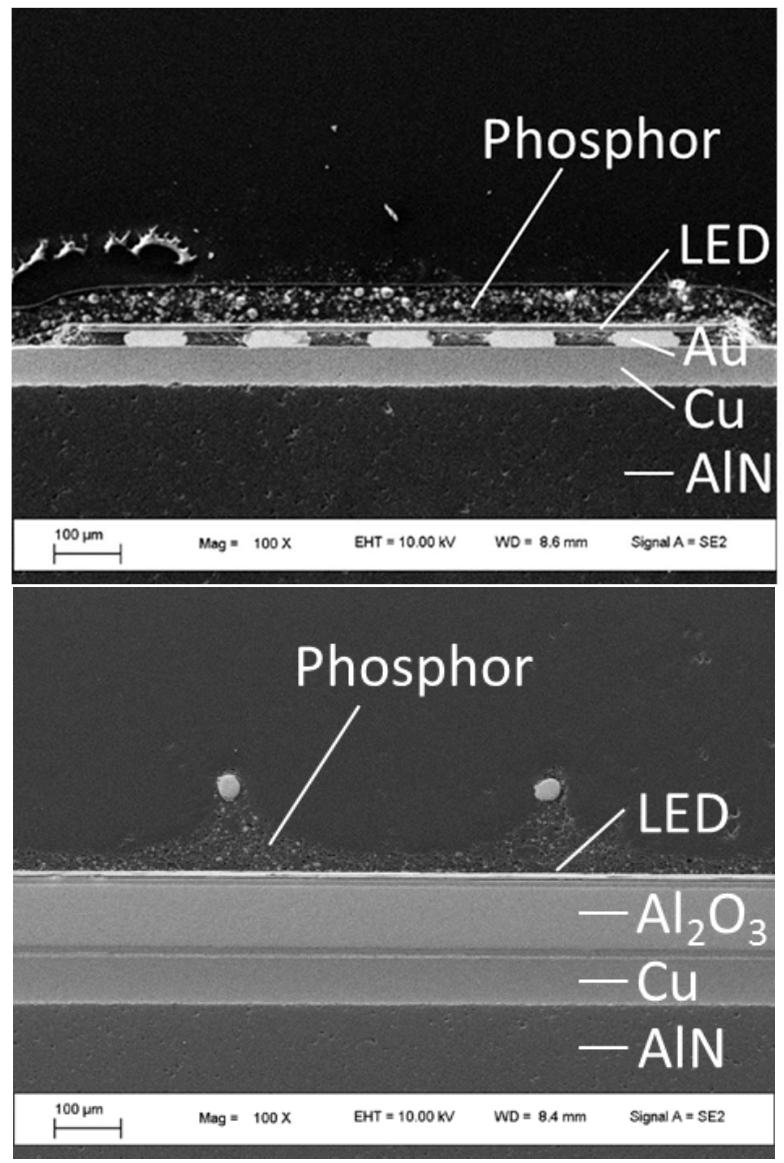

Fig. 1. Cross-sectional SEM images for each pcLED package: FC LED (top), C LED (bottom). 
The LED packages are mounted onto heatsinks and placed into environmental test chambers preset at $10 \%$ and $85 \%$ relative humidity (RH) at a temperature of $55^{\circ} \mathrm{C}$, with a bias current of $350 \mathrm{~mA}$. An integrated LED measurement system is used to evaluate the electrical-optical-thermal properties of the LED packages as shown in Fig. 2. The system consists of a Labsphere 20" integrating hemi-sphere system, a Keithley 2602A Source Meter, a Mentor Graphics T3ster, and a Peltierbased temperature controller (TEC). The LED is placed onto a temperature-controlled cold plate with a temperature tolerance of $+/-0.1^{\circ} \mathrm{C}$. The photometric properties of the emitted light output are measured using a spectro-radiometer. To ensure traceable optical measurements, reference lamp calibration and absorption correction are conducted prior to each measurement. For thermal measurements, the transient thermal response of the LED package is estimated using the electrical forward voltage $V_{F}$ method. A cooling measurement mode is adopted and the total radiant flux emitted from the LED is taken into consideration for the calculation of real thermal resistance, $\mathrm{R}_{\text {th-real }}$ to take into account the actual emitted optical power. The time-dependent behaviour of the heat flow path in the LED packages is analyzed using the structure function-based evaluation of the thermal transient measurements. An electrical I-V measurement is conducted before and after the optical-thermal measurements to ensure that the LED device did not degrade after each measurement.

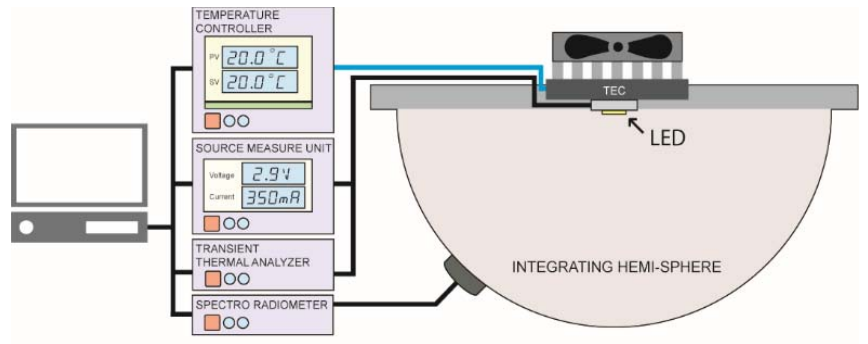

Fig. 1. Integrated LED measurement system consisting of an integrating hemi-sphere, a Peltier-based temperature controller, a source measure unit and a transient thermal tester.

\section{Results and Discussion}

Fig. 3 shows the optical degradation of both conventional (C) and flip-chip (FC) bonded LEDs. The optical characteristics observed is related to both the chip and package degradation. At the chip level, the decrease in radiant power is attributed to increased non-radiative recombination processes due to the generation of defects in the active region. This in turn leads to increased heat losses and higher junction temperatures. At the package level, dissolution of the phosphor and discoloration of encapsulation materials $[3,10]$ could exacerbate the optical output degradation.

The conventional bonded LEDs exhibit a high external quantum efficiency at both relative humidity $(\mathrm{RH})$ levels and the radiant power reduces steadily with time at $85 \% \mathrm{RH}$. However, a more stable radiant power output at $10 \% \mathrm{RH}$ is observed over the test period. The results indicated that the higher relative humidity results in a higher amount of degradation. On the other hand, flip-chip bonded LEDs have a lower overall radiant power output compared to the conventional LEDs and degrade steadily over time at both humidity levels. The lower overall optical output of the FC LEDs are due to the denser phosphor concentration (Fig. 1) causing higher light scattering and absorption losses in the phosphor layer, leading to higher extraction losses for the FC LEDs. Research on this impact of the phosphor layer on the optical output has been reported elsewhere by this research team [11].
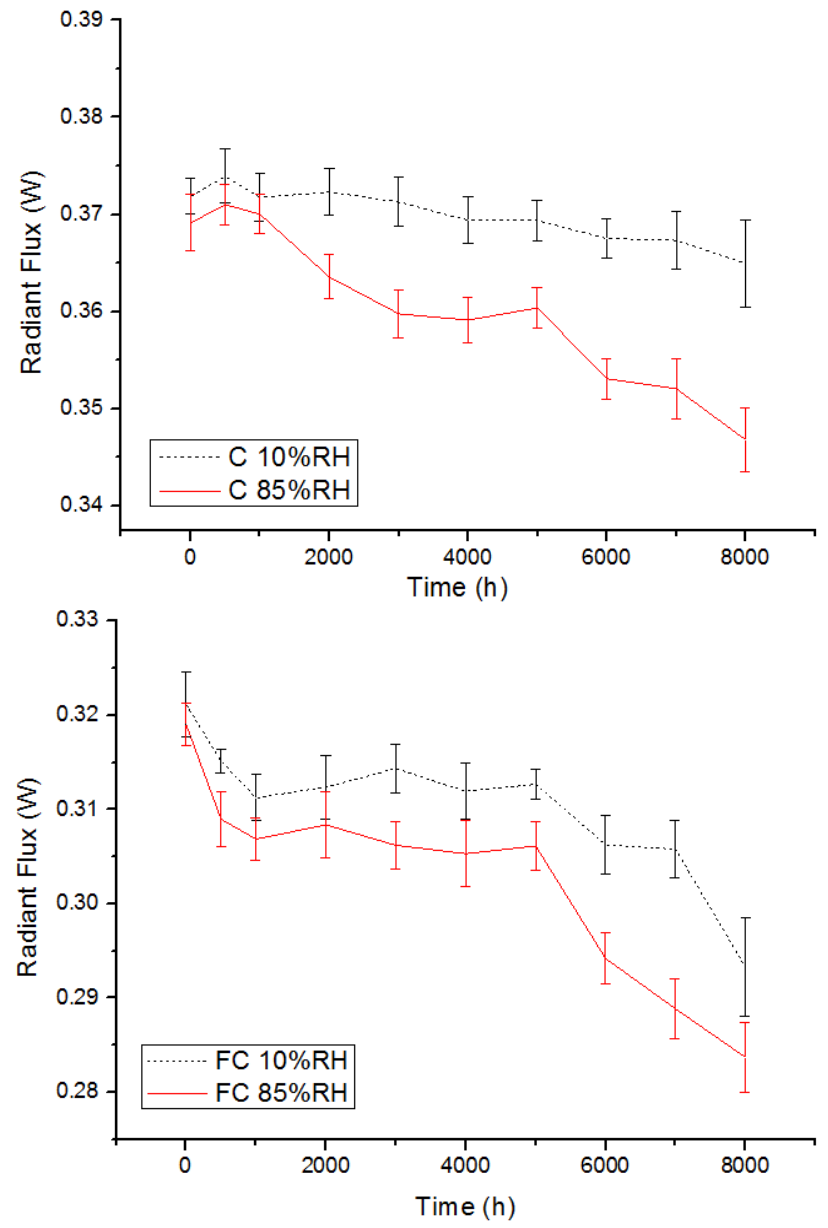

Fig. 3. Radiant flux degradation of C (top) and FC (bottom) LEDs at both $10 \%$ and $85 \% \mathrm{RH}$.

In a phosphor converted LED package, the light emitted from the package is determined by both the LED device and the phosphor layer. To further examine the light extraction losses contributed by the phosphor layer and the LED, the blue (LED) and yellow (phosphor) light outputs are computed separately as yellow-blue (Y-B) ratio. Fig. 4 shows the comparison of Y-B ratio of both types of LEDs at $10 \% \mathrm{RH}$ and $85 \% \mathrm{RH}$. For both $\mathrm{C}$ and FC LEDs, the samples under $85 \% \mathrm{RH}$ demonstrate higher Y-B ratio than $10 \% \mathrm{RH}$, indicating a higher yellow emission than blue emission at higher humidity. This suggests that LED degradation may be more pronounced than phosphor degradation at the higher humidity level. It is observed that the Y-B ratio of C LEDs has a lower rate of increase over the test period versus the FC LEDs. The change in Y-B ratio over 8000 hours of the $\mathrm{C}$ LEDs is about double at $85 \%$ RH compared to $10 \%$ RH. On the other hand, the Y-B of FC LEDs increases 3.2 times at 
$85 \% \mathrm{RH}$ compared to $10 \% \mathrm{RH}$. This observation is symptomatic of LED chip degradation as the predominant failure mechanism in the FC LEDs. The Y-B changes, together with other key parametric changes over the test period are summarized in Table 1.
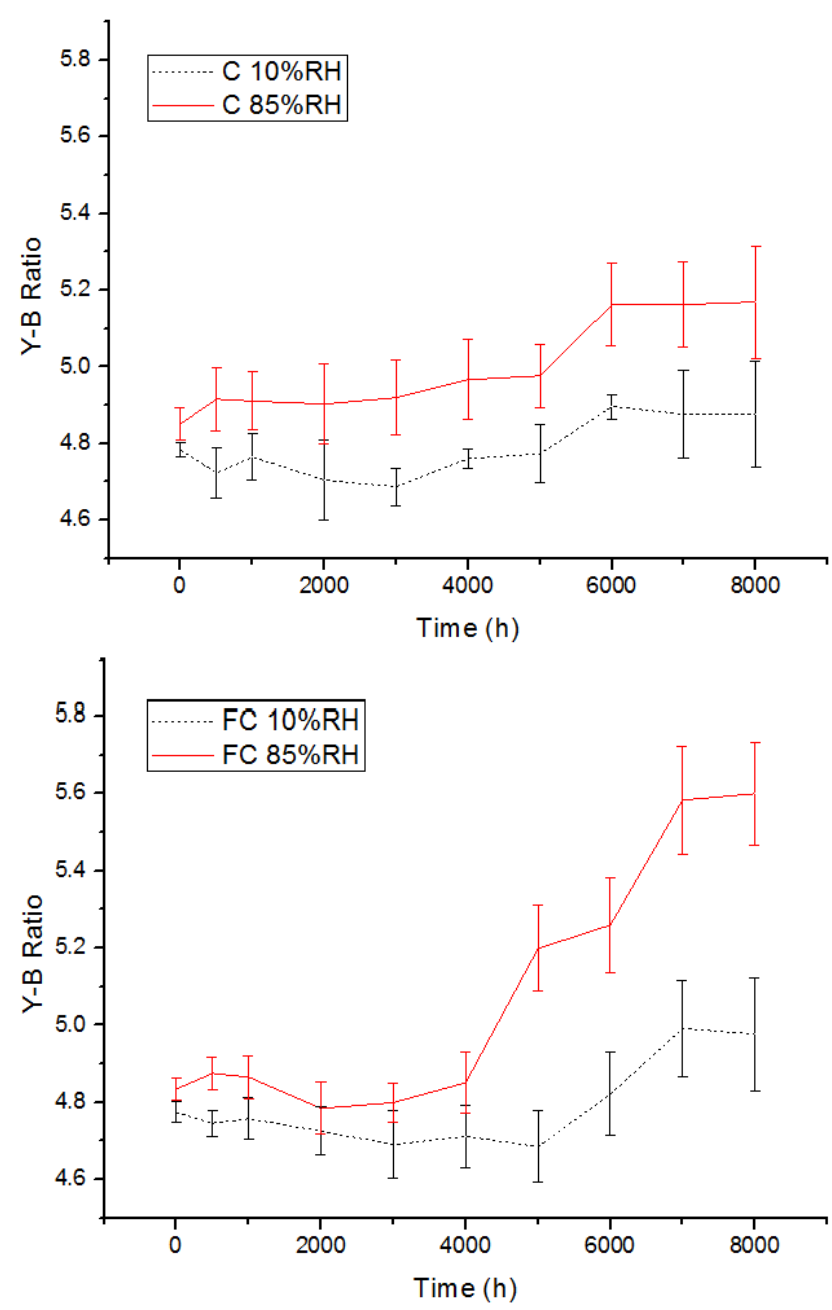

Fig. 4. Y-B ratio of $\mathrm{C}$ (top) and $\mathrm{FC}$ (bottom) LEDs at both $10 \%$ and $85 \% \mathrm{RH}$.

To provide further insight into the Y-B ratio trends, the spectral power distribution (SPD) characteristics of the LEDs are obtained as shown in Fig. 5. Both sets of LEDs exhibit a blue peak emission of $450 \mathrm{~nm}$. The C LEDs exhibit both higher blue and yellow intensity throughout the test period compared to the FC LEDs. As the humidity level increases, the intensity of both blue and yellow light decreases. However, while the yellow and blue intensity of the C LEDs degrade steadily over the test period, the blue intensity of the FC LEDs degrades more significantly at $85 \% \mathrm{RH}$ compared to $10 \% \mathrm{RH}$.
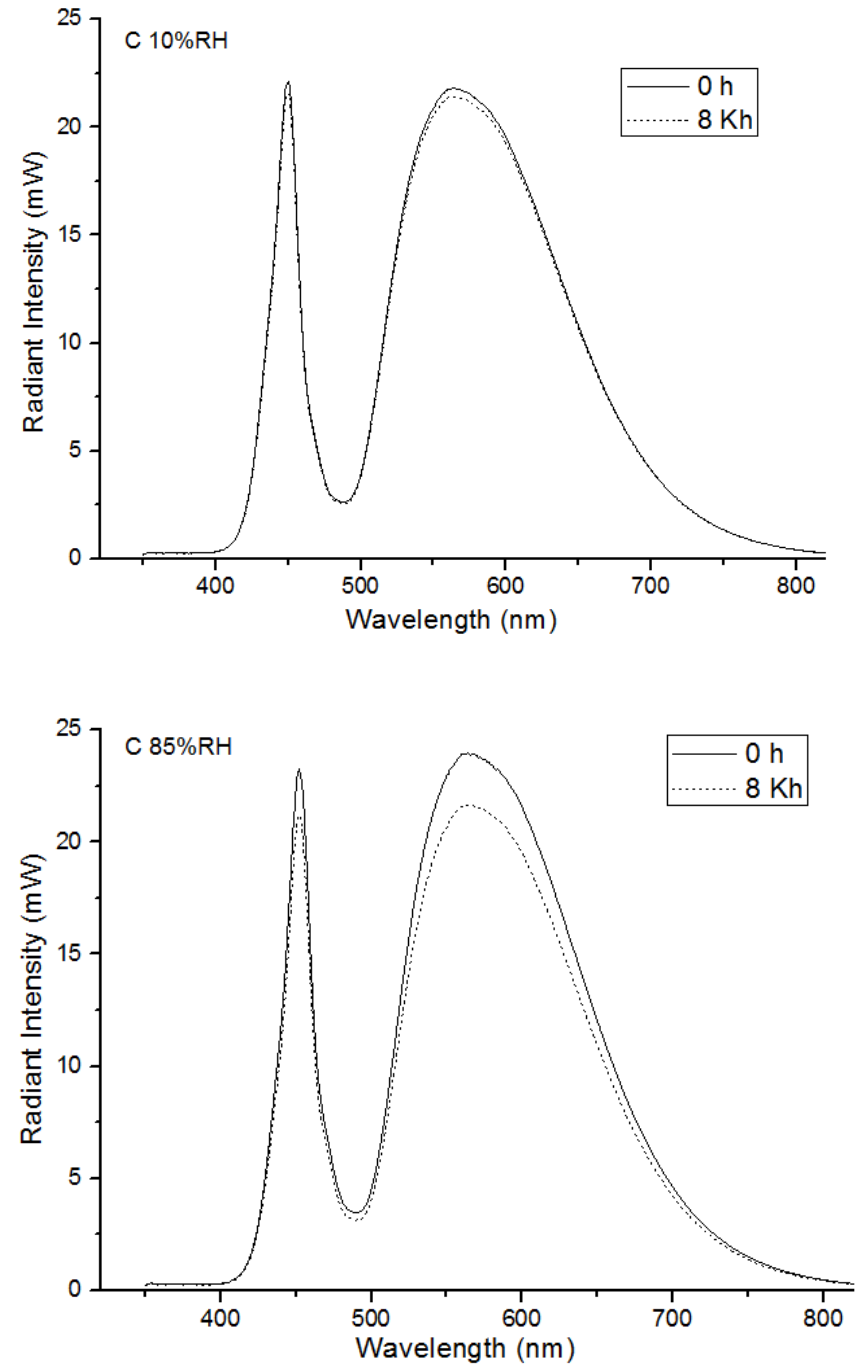

Fig. 5(a). Spectral power distribution of C LED at 10\% RH (top) and $85 \%$ RH (bottom) 

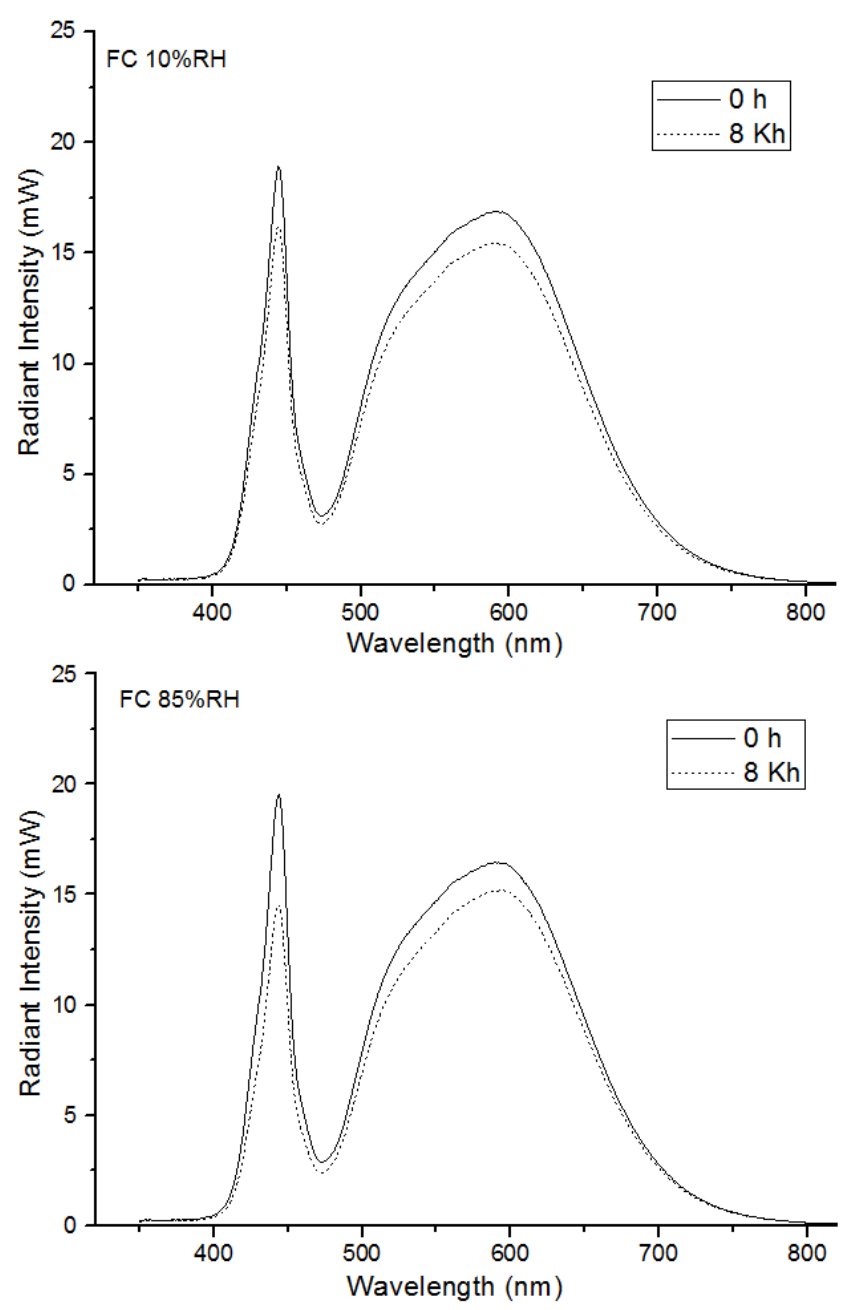

Fig. 5(b). Spectral power distribution of FC LED at 10\% RH (top) and $85 \%$ RH (bottom)

While the decrease of both blue and yellow emission in the LEDs are related to both chip failure and phosphor degradation, the more pronounced reduction in blue over yellow intensity for the FC LEDs at $85 \% \mathrm{RH}$ may indicate chip failure as a primary failure mechanism. In addition, the increase in series resistance $\mathrm{R}_{S}$ at $85 \% \mathrm{RH}$ (Table 1) compared to at $10 \% \mathrm{RH}$ for both LEDs indicates degradation of the electrical properties of the devices due to increased resistivity of the contact and semiconductor material and to the degradation of the properties of the ohmic contacts [12].

The Y-B characteristics correspond to a significantly higher increase in the forward voltage of $0.289 \mathrm{~V}$ for the FC LEDs at $85 \% \mathrm{RH}$ compared to $0.164 \mathrm{~V}$ at $10 \% \mathrm{RH}$. In contrast, the change in forward voltage for the C LEDs at $85 \%$ $\mathrm{RH}$ is only $0.147 \mathrm{~V}$ and the voltage change at $10 \% \mathrm{RH}$ is $0.132 \mathrm{~V}$. The increase in forward voltage could be caused by the increasing series resistance over the test period. The higher voltage change for the FC LEDs implies higher power output which should translate to higher heat generation compared to the C LEDs.
TABLE 1: PARAMETRIC Changes FOR C AND FC LEDS OVER 8000 HOURS AT $10 \%$ RH AND $85 \%$ RH

\begin{tabular}{|l|c|c|c|c|c|c|}
\hline \multirow{2}{*}{ Parameter } & \multicolumn{2}{|c|}{$\begin{array}{l}\text { Change over 8Kh } \\
\text { @ } 10 \% \mathrm{RH} \text { (a) }\end{array}$} & \multicolumn{2}{|c|}{$\begin{array}{c}\text { Change over 8Kh } \\
\text { @ } 85 \% \mathrm{RH} \text { (b) }\end{array}$} & \multicolumn{2}{|c|}{$\begin{array}{c}\text { Difference } \\
\text { between RH } \\
\text { [(b)-(a) }\end{array}$} \\
\cline { 2 - 7 } & $\mathrm{C}$ & $\mathrm{FC}$ & $\mathrm{C}$ & $\mathrm{FC}$ & $\mathrm{C}$ & $\mathrm{FC}$ \\
\hline $\mathrm{R}_{\mathrm{S}}(\Omega)$ & 0.261 & 0.325 & 0.362 & 0.589 & 0.101 & 0.264 \\
\hline Y-B ratio & 0.152 & 0.252 & 0.294 & 0.808 & 0.142 & 0.556 \\
\hline $\begin{array}{l}\text { Forward } \\
\text { voltage @350 } \\
\text { mA (V) }\end{array}$ & 0.132 & 0.164 & 0.147 & 0.289 & 0.015 & 0.125 \\
\hline $\begin{array}{l}\text { Temperature } \\
\left.\text { rise ( }{ }^{\circ} \mathrm{C}\right)\end{array}$ & 5.8 & 1.8 & 11.1 & 3.5 & 5.3 & 1.7 \\
\hline $\begin{array}{l}\text { Thermal } \\
\begin{array}{l}\text { Resistance } \\
(\mathrm{K} / \mathrm{W})\end{array}\end{array}$ & 6.0 & 1.0 & 11.0 & 2.5 & 5.0 & 1.5 \\
\hline
\end{tabular}

However this increase in heat generation for the FC LEDs over the C LEDs is not reflected in the graphs showing the temperature rise (Fig. 6). At 10\% RH, the FC LEDs were observed to have a minimum to maximum temperature rise of $1.8{ }^{\circ} \mathrm{C}$, while at $85 \% \mathrm{RH}$, the change in temperature rise was $3.5{ }^{\circ} \mathrm{C}$. The corresponding temperature rises for the $\mathrm{C}$ LEDs at $10 \% \mathrm{RH}$ and $85 \% \mathrm{RH}$ were observed to be $5.8{ }^{\circ} \mathrm{C}$ and 11.1 ${ }^{\circ} \mathrm{C}$ respectively. It is postulated that heat accumulation can be reduced significantly through the LED die-bonding configuration. A flip-chip bonded LED dissipates the heat generated in the GaN LED effectively, and exhibits a decrease in temperature rise compared to C LEDs.
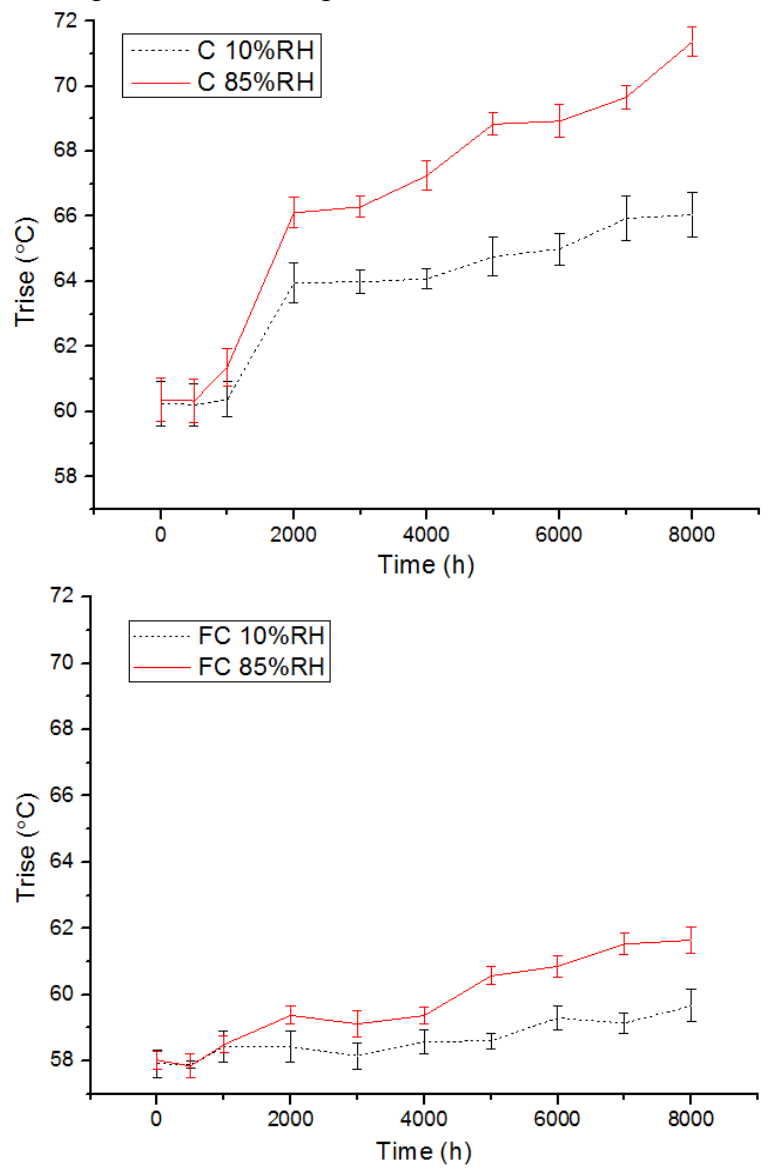

Fig. 6. Temperature rise of C (top) and FC (bottom) LEDs at both $10 \%$ and $85 \%$ RH. 
The bulk of the heat generated in the LED device is dissipated through the LED package, allowing for possible heat flow paths. To analyze the thermal response in the LED package, structure function evaluation of the two types of LEDs were computed for both $\mathrm{RH}$ levels (Fig 7). The differential structure function is the derivative of the cumulative thermal capacitance with respect to the cumulative thermal resistance [13]. The recorded cooling curves in the transient thermal measurements from the T3ster were evaluated to extract the thermal characteristics. The differential structure functions obtained from the evaluation were used to identify the partial thermal resistance of different sections in the heat conductance path. At both $10 \% \mathrm{RH}$ and $85 \% \mathrm{RH}$, the $\mathrm{C}$ LEDs show a greater increase in thermal resistance compared to the FC LEDs over the test period. This is consistent with higher change in temperature rise seen for the C LEDs. The smaller thermal resistance change seen in the FC LEDs could be attributed to the flip-chip die-bonding which allows for effective heat transfer onto the AIN substrate. Consequently, the FC LEDs have lower thermal resistances of $73 \mathrm{~K} / \mathrm{W}$ at $10 \% \mathrm{RH}$ and $76 \mathrm{~K} / \mathrm{W}$ at $85 \% \mathrm{RH}$ compared to the $\mathrm{C}$ LEDs which have higher thermal resistances of $82 \mathrm{~K} / \mathrm{W}$ at $10 \% \mathrm{RH}$ and $85 \mathrm{~K} / \mathrm{W}$ at $85 \% \mathrm{RH}$ respectively.

For the C LEDs, heat generated by the GaN LED device is transferred to the AlN substrate through the thick sapphire material. Due to the poor intrinsic thermal conductivity of the sapphire material $\left(\mathrm{KAl}_{2} \mathrm{O}_{3} \sim 46 \mathrm{~W} / \mathrm{m} \cdot \mathrm{K}\right)$, there is significant heat accumulation within the GaN LED device. Due to this, the C LEDs exhibit a higher temperature increase compared to the FC LEDs. On the other hand, flip-chip bonding allows effective heat transfer onto the AIN substrate due to the high thermal conductivity of the gold bumps $\left(\mathrm{K}_{\mathrm{Au}} \sim 318 \mathrm{~W} / \mathrm{m} \cdot \mathrm{K}\right)$. The heat generated by the FC LED is transferred effectively to the AlN substrate. Hence, the high optical loss in flip-chip bonded pcLEDs does not cause any significant changes to its thermal paths and merely exhibits a shift of partial thermal resistances. Almost all the heat fluxes generated in the LED is dissipated through the Au bumps and this demonstrates the fact that the packaging architecture has a significant influence on the temperature rise and thermal resistance of the package.
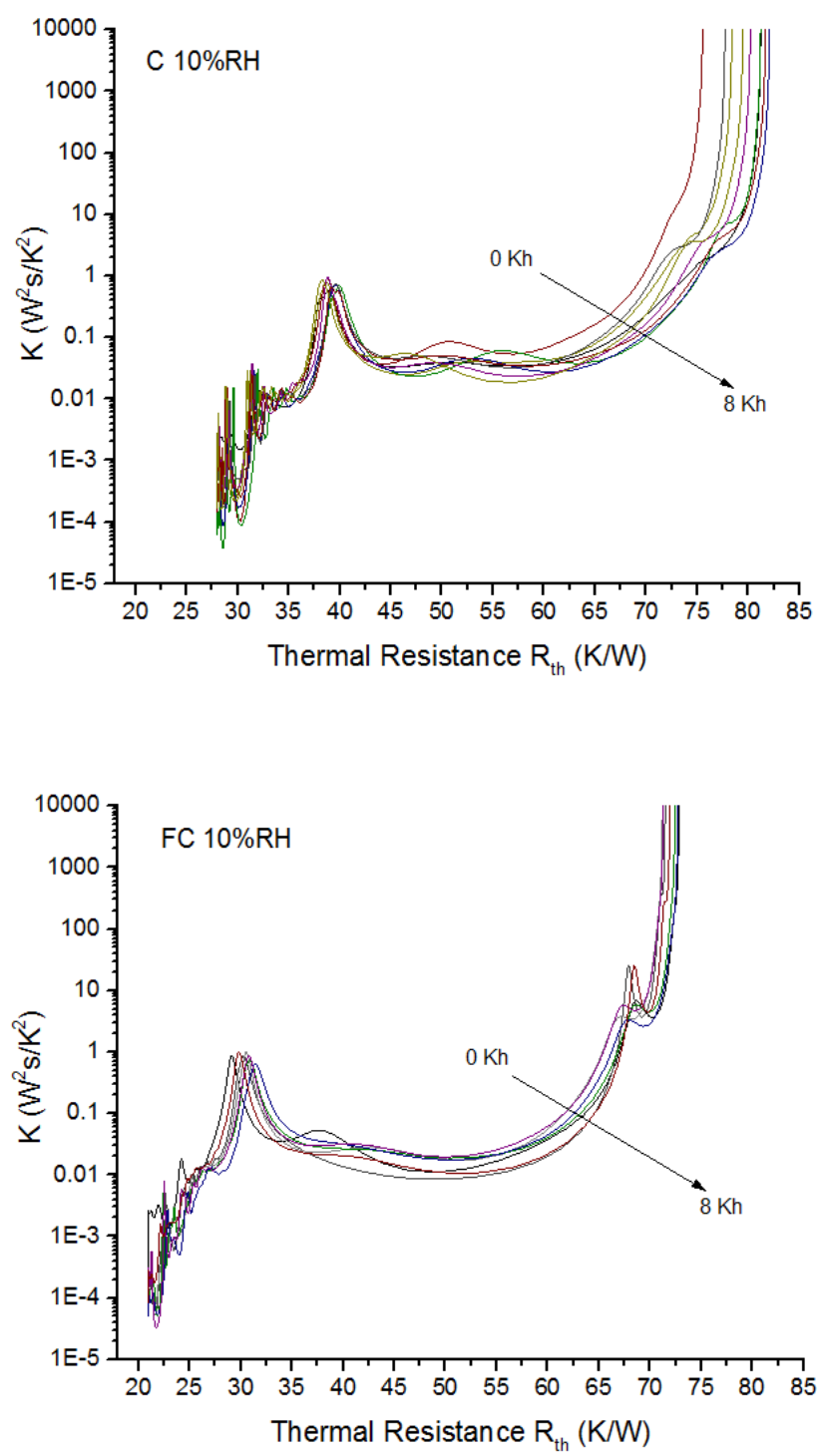

Fig. 7(a). Derivative Structure function evaluation of C (top) and FC (bottom) LEDs at $10 \% \mathrm{RH}$. 

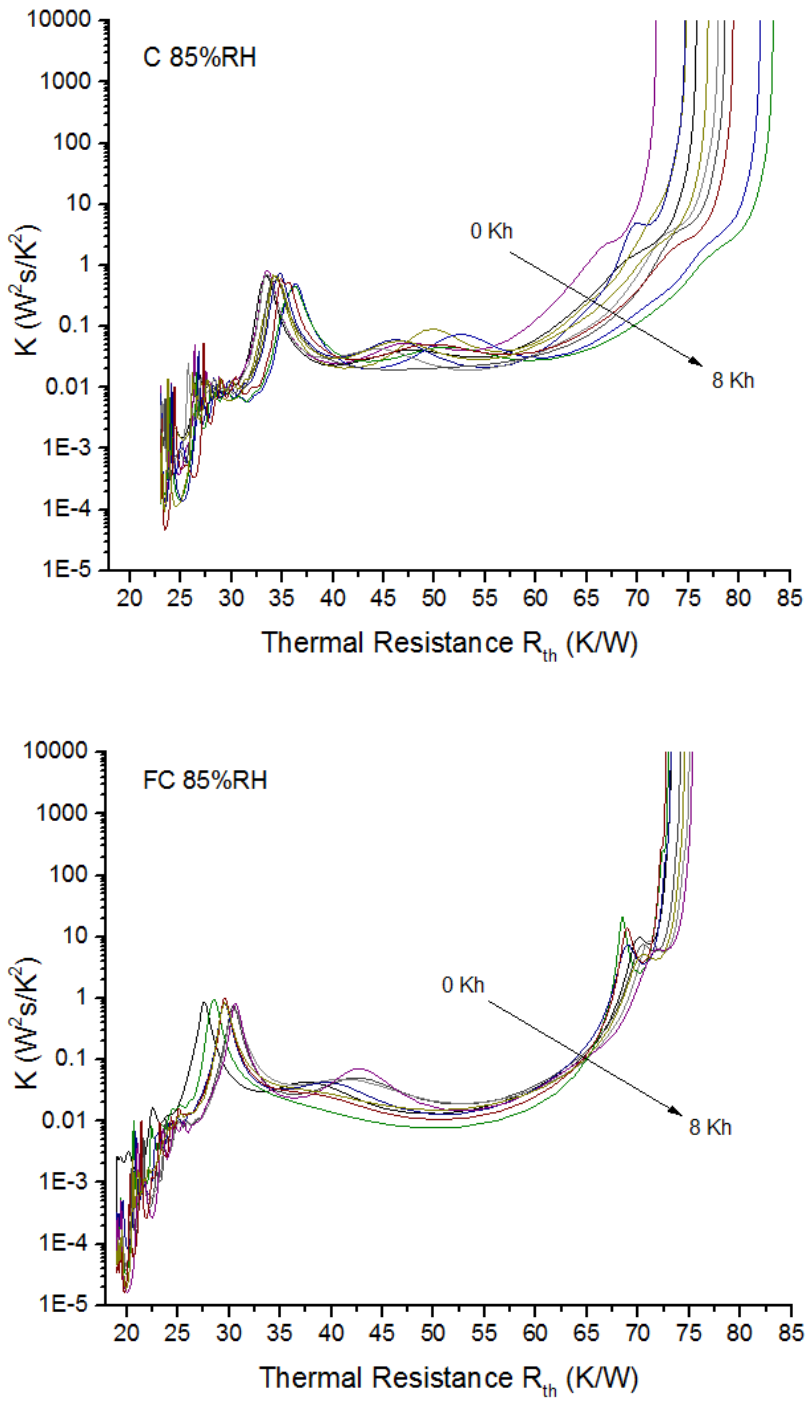

Fig. 7(b). Derivative Structure function evaluation of $\mathrm{C}$ (top) and FC (bottom) LEDs at $85 \%$ RH.

\section{Conclusions}

In this work, two sets of $1 \mathrm{~W}$ rated pcLED packages with different die-bonding configurations (flip-chip or conventional) white LEDs, were subjected to humidity stress tests of $10 \%$ and $85 \%$ relative humidity over a period of 8000 hours using an injection current of $350 \mathrm{~mA}$. Degradation of optical output power was observed, attributable to the generation of defects in the active region at the chip level and deterioration of phosphor or encapsulation materials at the package level. Detailed physical failure analysis is currently underway to supplement these results and will be reported subsequently. The flip-chip samples show higher Y-B ratio increase compared to the conventional samples, indicating lower blue light intensity over the test period, thereby suggesting that chip degradation may be more pronounced for the flip-chip batch compared to the conventional one. This induces heat accumulation and increases the temperature and thermal resistance in the LED package. However, the temperature rise and thermal resistance can be reduced with a flip-chip die-bonding configuration where the heat generated in the LED chip is dissipated effectively onto the AIN substrate. The results show that while the conventionally bonded samples exhibit better photometric and colorimetric performance, the flip-chip bonded LEDs show superior heat dissipating capabilities. As the thermal resistance of the LED package varies with different humidity levels, there is a need to specify the conditions of humidity in data sheets as LED manufacturers routinely specify a universal thermal resistance value under a fixed operating condition.

\section{References}

1. Singh, P. and C.M. Tan, A review on the humidity reliability of high power white light LEDs. Microelectronics Reliability, 2016. 61: p. 129-139.

2. Xiaobing, L., W. Bulong, and L. Sheng, Effects of Moist Environments on LED Module Reliability. IEEE Transactions on Device and Materials Reliability, 2010. 10(2): p. 182-186.

3. Tan, C.M., et al., Analysis of humidity effects on the degradation of high-power white LEDs. Microelectronics Reliability, 2009. 49(9): p. 1226-1230.

4. Hu, J., L. Yang, and M. Whan Shin, Mechanism and thermal effect of delamination in light-emitting diode packages. Microelectronics Journal, 2007. 38(2): p. 157-163.

5. $\mathrm{Hu}$, J., L. Yang, and M.W. Shin. Thermal effects of moisture inducing delamination in light-emitting diode packages. in Asia Pacific Optical Communications. 2006. International Society for Optics and Photonics.

6. Pei, Q., L. Qingqian, and Y.C. Chan. Thermal analysis of high brightness flip-chip LED packages. in Electronics Packaging Technology Conference (EPTC), 2011 IEEE 13th. 2011.

7. Wang, C.-P., et al., Analysis of thermal characteristics and mechanism of degradation of flip-chip high power LEDs. Microelectronics Reliability, 2012. 52(4): p. 698-703.

8. Wierer, J.J., et al., High-power AlGaInN flip-chip light-emitting diodes. Applied Physics Letters, 2001. 78(22): p. 3379-3381.

9. Chan, K.H., et al., The Effects of Temperature and Relative Humidity on the Viability of the SARS Coronavirus. Advances in Virology, 2011. 2011.

10. Zhou, S., et al. Evaluation of GaN-based blue light emitting diodes based on temperature/humidity accelerated tests. in Electronic Packaging Technology \& High Density Packaging (ICEPT-HDP), 2010 11th International Conference 2010 .

11. Law, T.K., et al., Implications of Phosphor Coating on the Thermal Characteristics of Phosphor-Converted White LEDs. IEEE Transactions on Device and Materials Reliability, 2016. PP(99): p. 1-1.

12. Meneghini, M., et al., A review on the reliability of GaN-based LEDs. IEEE Transactions on Device and Materials Reliability, 2008. 8(2): p. 323-331.

13. Székely, V., A new evaluation method of thermal transient measurement results. Microelectronics Journal, 1997. 28(3): p. 277-292. 